\title{
New Mechanisms of Tumor-Associated Macrophages on Promoting Tumor Progression: Recent Research Advances and Potential Targets for Tumor Immunotherapy
}

\author{
Qiujun Guo, ${ }^{1,2}$ Zhichao Jin, ${ }^{1}$ Yuan Yuan, ${ }^{1,2}$ Rui Liu, ${ }^{1}$ Tao Xu, ${ }^{3}$ Huamin Wei, ${ }^{1}$ Xinyao Xu,,${ }^{1,2}$ \\ Shulin He, ${ }^{1,2}$ Shuntai Chen, ${ }^{1,2}$ Zhan Shi, ${ }^{4}$ Wei Hou, ${ }^{1}$ and Baojin Hua ${ }^{1}$ \\ ${ }^{1}$ Department of Oncology, Guanganmen Hospital, China Academy of Chinese Medicine Sciences, No. 5 Beixiange, \\ Xicheng District, Beijing 100053, China \\ ${ }^{2}$ Beijing University of Chinese Medicine, No. 11 North Third Ring Road East, Chaoyang District, Beijing 100029, China \\ ${ }^{3}$ Department of Oncology, Xiyuan Hospital, China Academy of Chinese Medicine Sciences, No. 1 Playground Road, \\ Haidian District, Beijing 100091, China \\ ${ }^{4}$ Institute of Basic Research in Clinical Medicine (IBRCM), China Academy of Chinese Medicine Sciences, \\ No. 16 Dongzhimen Nanxiaojie, Dongcheng District, Beijing 100700, China \\ Correspondence should be addressed to Zhan Shi; drshizhan@126.com and Baojin Hua; dr.huabaojin@hotmail.com
}

Received 20 June 2016; Accepted 26 September 2016

Academic Editor: Eyad Elkord

Copyright (C) 2016 Qiujun Guo et al. This is an open access article distributed under the Creative Commons Attribution License, which permits unrestricted use, distribution, and reproduction in any medium, provided the original work is properly cited.

\begin{abstract}
The majority of basic and clinical studies have shown a protumor function of tumor-associated macrophages (TAMs), which represent a large proportion of matrix cells. TAMs promote tumorigenesis, and their number is related to the malignancy degree and poor prognosis of many kinds of tumors. Macrophage plasticity makes it possible to change the tumor microenvironment and remodel antitumor immunity during cancer immunotherapy. Increasing numbers of studies have revealed the effects of TAMs on the tumor microenvironment, for example, via promotion of tumor growth and tumorigenesis and through an increase in the number of cancer stem cells or via facilitation of angiogenesis, lymphangiogenesis, and metastasis. Investigators also proposed tumor-immunological treatments targeting TAMs by inhibiting TAM recruitment and differentiation, by regulating TAM polarization, and by blocking factors and pathways associated with the protumor function of TAMs. This comprehensive review presents recent research on TAMs in relation to prediction of poor outcomes, remodeling of the tumor immune microenvironment, and immunological targeted therapies.
\end{abstract}

\section{Introduction}

Macrophages are differentiated cells of the mononuclear phagocytic lineage. They are heterogeneous cells with distinct functions and respond differently to various microenvironmental signals and thus have distinct functions. Macrophages-derived from hematopoietic stem cells in bone marrow or from progenitor cells in the embryonal yolk sac-differentiate into two distinct types of macrophages $(\mathrm{M} \phi)$, classically activated or M1 M $\phi$ ("killing" phenotype) and alternatively activated or M2 M $\phi$ ("healing" phenotype). Tumor-associated macrophages (TAMs) may represent up to $50 \%$ of the tumor mass, and most TAMs have the M2 phenotype due to the signals in the tumor microenvironment, such as IL-4 and TGF- $\beta[1,2]$. As generally accepted at present, TAMs play a key role in tumorigenic processes ranging from tumor initiation to acceleration of tumor progression and metastasis.

\section{TAMs Lead to a Poor Clinical Prognosis and Promote Progression of Various Tumors}

2.1. TAMs Can Be Considered a Biomarker of Poor Prognosis. TAMs provide a suitable microenvironment for tumor invasion and progression and contribute to the metastasis of tumor cells [3]. Researchers have shown that the existence of TAMs positively correlates with a poor clinical prognosis 
of various tumors, such as lung cancer, gastric cancer, gynecological tumors, and lymphomas (Table 1). Although there are a few exceptions to this association, such as colorectal carcinoma [4], these data still indicate that TAMs play an essential role in tumorigenesis; accordingly, targeting of TAMs may be a promising method of cancer treatment.

\subsection{TAMs Reduce the Curative Effect of Chemotherapy.} Chemotherapy is a conventional treatment modality for cancer patients. Although chemotherapies have strong effects on some kinds of tumors, such as small cell lung cancer [5] and lymphomas [6], the overall response rate is less than satisfactory for many tumors [7]. Furthermore, drug resistance, tumor recurrence, and metastasis make tumor treatment more difficult.

TAMs were found to help reduce the effects of chemotherapy. Infiltration by $\mathrm{CD} 8^{+}$and $\mathrm{CD} 163^{+}$TAMs is associated with a poor response to chemotherapy in patients with esophageal cancer $[8,9]$. TAMs are also recruited to the pancreatic tumor site and stimulate adenocarcinoma cells to express high levels of cytidine deaminase, which catabolizes the bioactive form of gemcitabine and reduces the sensitivity of cancer cells to chemotherapy [10]. Yang et al. [11] also found that TAMs can induce chemotherapeutic drug resistance of a breast tumor through the IL-10/STAT3/Bcl-2 signaling pathway, and another study showed that TAMs correlate with the resistance to tamoxifen, an endocrine therapy drug for postmenopausal breast cancer patients [12].

Epidermal growth factor receptor tyrosine kinase inhibitors (EGFR-TKIs) are novel treatments of lung cancer with EGFR mutation and have higher specificity and fewer side effects than traditional treatments; M2-polarized TAMs also correlate with the decreased responsiveness to EGFR-TKI treatment in patients with advanced lung adenocarcinoma [13].

\subsection{TAMs Cause an Unfavorable Outcome of Radiotherapy.} Radiotherapy (RT) is a localized therapy that is highly effective at killing primary tumor cells located within the field of the radiation beam. Despite sophisticated techniques for radiation delivery as well as the combination of radiation with chemotherapy, tumors can recur [14].

TAMs are abundantly recruited to tumors after irradiation and may modulate cancer cells' responses to therapy [15]. It has been suggested that macrophages may be involved in modulating the late effects of radiotherapy [16]. Various studies indicate a correlation between high TAM numbers and poor tumor responses to irradiation in mouse models [17]. Furthermore, Shiao et al. [18] reported that regrowth of mammary tumors after radiotherapy correlates with the influx of Th2-polarized macrophages. After tumor irradiation, DNA damage, cell death, and increased tumor hypoxia may promote production of VEGF, SDF-1, and CSF-1, resulting in the recruitment, infiltration, and retention of monocytes/macrophages within the tumor [14]. The recruited heterogeneous populations of TAMs release proangiogenic cytokines and metalloproteinases to promote blood vessel formation within the tumor [19]. Teresa Pinto et al. [20] also found that irradiated macrophages promote cancer cell invasiveness and cancer cell-induced angiogenesis. Besides, the abscopal effect may be a crucial factor in evaluation of the prognosis associated with radiotherapy, and TAMs can release secondary bystander signals and play a key role in the secondary bystander effect of photon irradiation [21]. Targeting TAMs or TAM-associated signaling to enhance the potency of radiotherapy has been similarly demonstrated in several other studies [14, 22-24]. Inhibition of TAM recruitment after radiotherapy by neutralizing CSF-1 or blocking CSF-1R kinase activity may significantly slow tumor regrowth [15].

\section{TAMs Remodel the Tumor Microenvironment}

3.1. TAMs Ensure an Immunosuppressive Tumor Microenvironment. The tumor immune microenvironment is mainly formed by such immune cells as macrophages, $\mathrm{T}$ lymphocytes, natural killer cells (NK cells), dendritic cells, neutrophils, and myeloid-derived suppressor cells (MDSCs) [47, 48]. As a kind of immunosuppressive cell subgroup, TAMs express chemokines and cytokines and contribute to the immunosuppressive tumor microenvironment (ITM) [49]. Chemokines (such as CCL5, CCL22, and CCL20) secreted by TAMs recruit regulatory $\mathrm{T}$ (Treg) cells, whereas cytokines (such as IL-10 and TGF- $\beta$ ) induce Treg cells. Besides, TAMs may inhibit the antitumor effect of tumor-infiltrating T cells and NK cells $[50,51]$ and promote ITM synergistically with MDSCs, tumor-associated dendritic cells, and neutrophils [47, 52-54]. TAMs may suppress T-cell function by secreting specific enzymes such as nitric-oxide synthase (NOS) and arginase (ARGI) $[55,56]$. In addition, TAMs express ligands for receptors called PD-1 and CTLA-4 (such ligands as PDL1 and B7-H1), which after activation suppress cytotoxic functions of T cells, NKT cells, and NK cells [57].

3.2. TAMs Promote Tumorigenesis. Cancer can be considered a nonresolving inflammatory disease. Up to $20 \%$ of all cancers arise in association with chronic inflammation, and almost all solid tumors contain inflammatory infiltrates [58]. TAMs, as the major immune cells in a tumor, have a broad impact on tumor initiation. Macrophages can play contrasting roles in cancer depending on their phenotype. M1-type macrophages have the potential to contribute to the earliest stages of neoplasia, whereas M2-type macrophages usually get involved after a tumor progresses and grows [59]. DNA damage is the major mechanism of tumorigenesis induced by inflammation. The free radicals produced by TAMs can lead to DNA damage, causing mutations that predispose the affected individual to cancer. An example of this macrophagemediated induction of tumorigenesis is that Crohn's disease dramatically increases the risk of colorectal cancer [60-63]. Besides, chronic infection with viruses such as hepatitis B virus in the liver or with bacteria like Helicobacter pylori in the stomach or continuous exposure to irritants such as asbestos in lungs is casually associated with cancer initiation.

3.3. TAMs Facilitate Metastasis. Metastasis is responsible for more than $90 \%$ of cancer mortality yet remains the least 


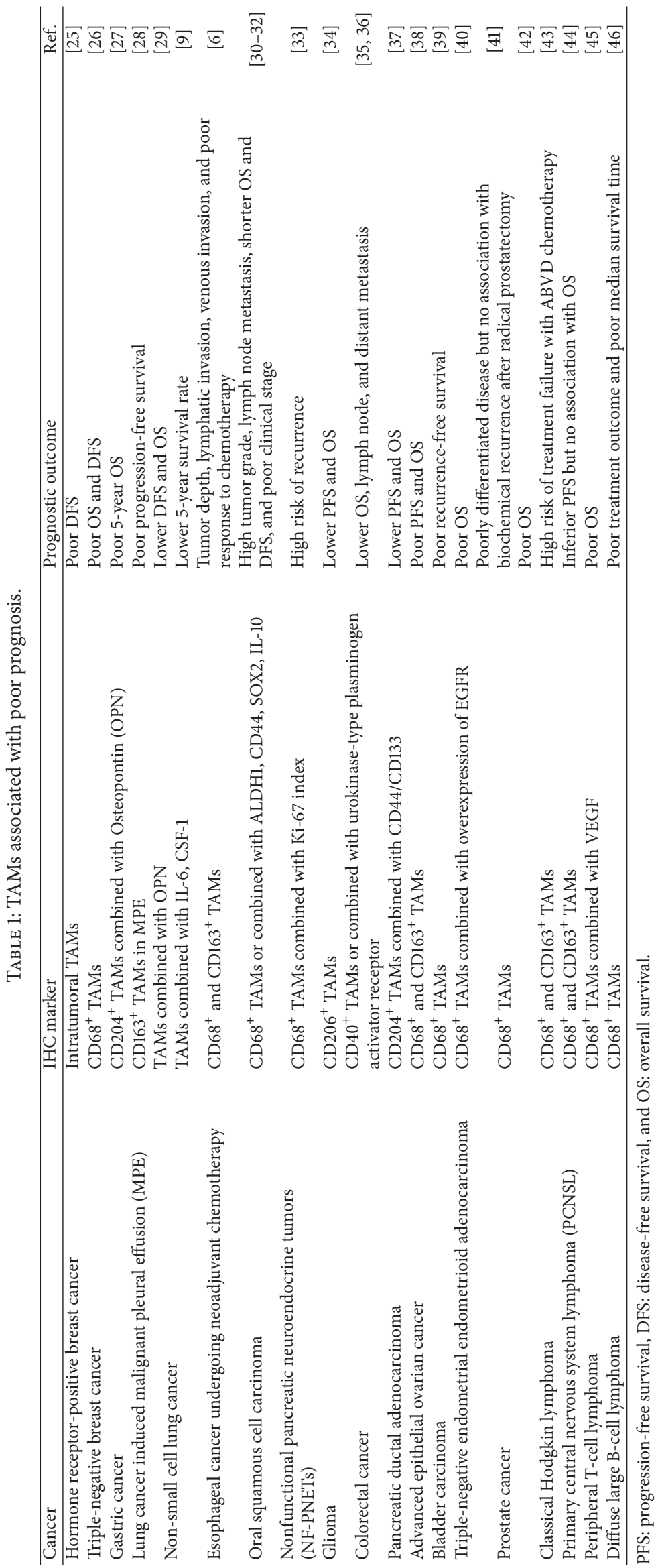


understood stage of tumor progression. TAMs have been shown to be key players in metastasis and mainly participate in several steps including epithelial-to-mesenchymal transition (EMT), local invasion and intravasation into the vasculature, transit through the circulatory system, extravasation and seeding in the premetastatic niche, and finally survival and growth at the metastatic site $[64,65]$. TAMs can promote progression and metastasis through the release of a variety of chemokines, inflammatory factors, and growth factors. It is reported that $\mathrm{CD} 68^{+} \mathrm{HLA}-\mathrm{DR}^{+}$TAMs in hepatocellular carcinoma (HCC) can promote migration of HCC cells via the NF- $\kappa$ B/FAK pathway [66]. Specifically, TAM-derived IL6 and IL- 8 enhance invasive activity of LoVo cells induced by PRL-3 in a KCNN4 channel-dependent manner [67].

In breast tumors, TAMs are recruited to pulmonary metastases by CCL2 and enhance extravasation, seeding, and persistent growth of tumor cells in part via expression of VEGF [68]. CCL2 synthesized by a tumor and stroma also triggers a prometastatic chemokine cascade (involving CCL3 signaling via CCR1) that is required for efficient metastasis [69]. Besides, TAMs binding to VCAM-1 expressed on breast cancer cells can promote tumor cell survival in lungs [70]. The bidirectional cross-talk between cancer cells and TAMs constitutes a microanatomic landmark, which is defined as the tumor microenvironment of metastasis (TMEM), whereas TMEM density is positively associated with the risk of distant organ metastases [71].

EMT is a process via which epithelial tumor cells lose epithelial features and gain mesenchymal phenotypes [72]. EMT is considered the key step via which tumor cells gain the greater capacity for invasiveness and metastasis. TAMs can strongly express many kinds of cytokines that can induce EMT, such as TGF- $\beta$ and IL-6 [73]. TNF- $\alpha$ secreted by TAMs has been shown to activate NF- $\kappa \mathrm{B}$-mediated transcription of Snaill and Zeb, which leads to diminished E-cadherin expression on tumor cells [74]. One report suggests that M2 macrophages may induce EMT by regulating TLR4/IL-10 signaling in pancreatic cancer cells [75]. TAMs may also secrete EGF-like ligands/factors that activate the EGFR pathway in cancer cells, thus promoting EMT, and this macrophageinduced EMT is significantly inhibited by treatment of A549 cancer cells with JWH-015 [76].

CCL18 released from TAMs can induce EndMT in endothelial cells to produce a different differentiated phenotype, which may lead to a loss of cell-cell junctions as well as enhanced invasiveness and migratory capacity [77]. These data confirm a strong cross-talk between macrophages and tumor progression, mainly through stimulation of EMT.

\subsection{TAMs Promote Angiogenesis and Lymphangiogenesis.} The growth and spread of neoplasms depend on angiogenesis and lymphangiogenesis in the tumor microenvironment. With neoplastic progression, increasing numbers of blood and lymphatic vessels provide supply channels for tumor tissues. On the other hand, it is reported that these mounting vessels provide a route for the lymph-nodal and distant metastases of tumor cells [78-81]. The infiltration by TAMs is associated with extensive angiogenesis, which contributes to a poor prognosis in primary cancers. Multiple factors are involved in angiogenesis: hypoxia, hyperosmosis, and proangiogenic factors such as vascular endothelial growth factor (VEGF), transforming growth factor beta (TGF- $\beta$ ), cyclooxygenase 2 (COX-2), platelet-derived growth factor (PDGF), epidermal growth factor (EGF), angiopoietins (Ang), and chemokines [82-86]. CCL18 released from TAMs can promote angiogenesis and tumor progression in breast cancer [70]. TAMs can also synthesize Wnt7b, which targets vascular endothelial cells by stimulating their production of VEGF resulting in the angiogenic switch [87]. One of the major angiogenesis-inducing factors, pro-matrix metalloproteinase-9 (proMMP-9), is supplied to the tumor microenvironment by TAMs [88]. It has been demonstrated that matrix metalloproteinase 9 (MMP-9) plays a crucial role in tumor angiogenesis and metastasis by turning on the angiogenic switch in avascular tumors and by mediating the development and maintenance of distinct neovascular networks sustaining tumor cell intravasation [89, 90]. Hypoxia causes a tumor microenvironment overexpressing hypoxiainducible factor $1 \alpha$ (HIF- $1 \alpha$ ), the primary transcription factor involved in homeostasis of oxygen concentration. HIF- $1 \alpha$ can activate transcription of genes encoding angiogenic growth factors and stimulating endothelial cells (ECs), thus leading to angiogenesis [75]. TAMs' localization to hypoxic tumor areas is controlled by the Sema3A/neuropilin-1 signaling axis, leading to plexinA1/plexinA4-dependent VEGFR1 activation and promotion of tumor growth and metastasis [91]. In addition, TAMs can increase the expression of HIF- $1 \alpha$ and promote endothelial-tube formation in colon cancer [92]. Besides, Laoui et al. found that hypoxia specifically lowers hypoxia-sensitive gene expression and angiogenic activity in the MHC-II ${ }^{\text {lo }}$ TAM subset instead of altering differentiation of the TAM population [93].

Coordination of the lymphatic microvascular network with the blood microvasculature is involved in normal physiological functions, such as local tissue fluid balance, tissue perfusion, and immune surveillance [94-96], and gives more weight to lymphangiogenesis during tumor growth and cancer metastasis [72]. A number of studies have revealed lymphatic microvessel density (LMVD) to be an independent prognostic factor for solid tumors [97-100]. Additionally, TAMs are reportedly involved in lymphangiogenesis in malignant tumors [101]. Zhang et al. found that TAMs in lung adenocarcinoma are associated with poor prognoses resulting from accelerated lymphangiogenesis and lymph node metastasis [102].

Tumor cells induce formation of lymphatic vessels via the lymphatic system metastasis through VEGF-C, VEGF$\mathrm{D}$, and other cytokines [103]. The expression of VEGF-C and VEGF-D by TAMs suggests that TAMs are intimately involved in the generation of tumor lymphatic vessels [104]. M2-polarized TAM infiltration of RLNs is significantly associated with nodal lymphangiogenesis, and node-infiltrating M2-polarized TAMs may facilitate nodal lymphangiogenesis via production of VEGF-C [105].

3.5. TAMs and Cancer Stem Cells (CSCs). CSCs or cancerinitiating cells are defined as a small subpopulation of cancer 
cells with the capacity for self-renewal and pluripotency. CSCs are necessary for initiation of new tumor growth at distant sites. Currently, many studies support the notion that CSCs, which have many features of stem cells, are responsible for the poor prognosis of patients by promoting tumor recurrence and metastasis $[106,107]$.

TAMs can regulate the plasticity of CSC phenotypes and functions. Recently, some of TAMs-CSCs interrelations were confirmed experimentally. TAMs were found to release milk-fat globule EGF-VIII, which activates the CSCspecific pathways-STAT3, Hedgehog, and Sonic-and strongly amplifies drug resistance and tumorigenicity of CSCs [108]. MFG-E8 and IL-6 from TAMs can also synergistically mediate tumorigenicity and drug resistance in subsets of CSCs including those in primary human tumors [109]. In experiments with murine mammary CSCs, Yang et al. reported that drug resistance of CSCs is associated with new EGFR/STAT3/Sox-2 paracrine signaling pathway activity that is realized via a complex interplay between CSCs and TAMs [110]. It is reported that TAMs have effects on gastric CSCs in omental milky spots and on lymph node micrometastasis, which is mainly mediated by activation of MCP-1, COX-2, PGE-2, IL-10, IFN- $\gamma$, and VEGF and by downregulation of IL-4, TGF- $\beta$, MMP-2, and MMP-9 [111]. It has been suggested TAMs may promote CSC-like properties via TGF- $\beta 1$-induced EMT and may advance the research on the prognosis of HCC [112]. Furthermore, microglia and brain TAMs serve as mediators of glioma stem-like cell (GSLC) properties, producing high levels of TGF- $\beta$, which makes GSLCs more invasive [113]. Direct cell-cell interactions of TAMs with CSCs via Thyl and Eph4A receptors have been reported to induce activation of NF- $\kappa \mathrm{B}$, which in turn sustains the CSCs state [114].

The functional and phenotypic heterogeneity of CSCs themselves in turn affects the pathophysiological activities of TAMs. It seems that active CSCs should be able to promote the M1-to-M2 conversion, induce formation of new vasculature via VEGF release, and build CSC-protective niches via tissue-repair pathways [2]. OCSCs may promote the M2 polarization of macrophages through the PPAR $\gamma / \mathrm{NF}-$ $\kappa \mathrm{B}$ pathway [115]. Chemoresistant CSCs may promote M2 macrophage differentiation through interferon-regulatory factor-5- (IRF5-) and macrophage-colony stimulating factor(M-CSF-) dependent mechanisms [116]. Chen et al. reported that embryonic stem cells can promote macrophage survival and M2-like activation, which are critically important for teratoma angiogenesis and development [117] (Figure 1).

TAMs can be considered a biomarker of poor prognosis and reduce the curative effect of chemotherapy and radiotherapy. In terms of mechanisms, TAMs may promote a tumor immunosuppressive microenvironment, tumorigenesis, angiogenesis, and lymphangiogenesis and can facilitate metastasis.

\section{Treatments Targeting TAMs}

Immunotherapy acts in a fundamentally different way in comparison with classical therapies. Rather than destroying tumor cells directly, immunotherapy promotes tumor cell killing via the immune response of the host. This result can be achieved directly via the main effectors of the immune system, such as macrophages. Evidence reviewed by Mills et al. indicates that modulation of macrophage responses is a breakthrough that will facilitate successful immunotherapy [118]. In a sorafenib-resistant tumor model, photoimmunotherapy targeting TAMs was found to inhibit the tumor growth and metastasis [119]. Therefore, TAMs have become promising therapeutic targets for cancer treatment. In recent years, many researchers focused on cancer immunotherapy related to TAMs (Table 2).

4.1. Killing of TAMs or Inhibition of TAM Recruitment and Differentiation. Strategies to deplete TAMs have been successful in experimental settings and are now considered a promising therapeutic approach in the clinic [120]. The current approach to targeting of TAMs that has shown efficacy is inhibition of CSF-1/CSF-1R signaling because this axis is required for macrophage survival [121, 122]. For instance, targeting of CSF-1/CSF-1R alters macrophage polarization and blocks glioma progression [123]. RG7155, a monoclonal antibody that inhibits CSF-1 receptor (CSF-1R), can provide significant clinical benefits and offer a therapeutic option other than surgical treatment to patients with a diffuse-type giant cell tumor (Dt-GCT) [124].

An RNA aptamer that blocks the murine or human IL-4 receptor- $\alpha$ (IL $4 \mathrm{R} \alpha$ or CD124) can preferentially target TAMs and unexpectedly promote their elimination, an effect that is associated with an increased number of tumor-infiltrating $\mathrm{T}$ cells and a reduction in tumor growth [125]. Allavena et al. have recently demonstrated that trabectedin, a licensed and commercially available anticancer agent, is selectively toxic to TAMs because of activation of caspase 8-dependent apoptosis $[126,127]$. Membrane-permeating drugs can also induce apoptosis in macrophages; this effect may be exploited for the depletion of TAMs [128]. Inhibiting recruitment of macrophages to neoplastic lesions is one of the therapies targeting TAMs. The antitumor agent dequalinium-14 in addition to its antitumor effect can reduce macrophage motility, inhibit macrophage infiltration of irradiated tumors, and reduce the extent of metastasis in locally irradiated mice [14]. Besides, TAMs derive from monocytes and have the capacity for differentiation; therefore, a combination therapy blocking differentiation may be required for effective targeting of these cells [129]. Interferon $\gamma$ may induce recruitment of monocytes/macrophages into the tumor microenvironment but inhibits their differentiation into TAMs in vivo; this effect may reduce the concentration of VEGF and angiogenesis in a tumor [130].

4.2. Regulation of TAM Polarization. TAMs are typically designated as "alternatively activated" noninflammatory M2type macrophages, in contrast to the "classically activated" inflammatory M1 type. TAMs coexist with tumors and function as an accomplice in the promotion of tumor progression, especially after being programmed and polarized into a proangiogenic/immunosuppressive (M2-like) phenotype by 

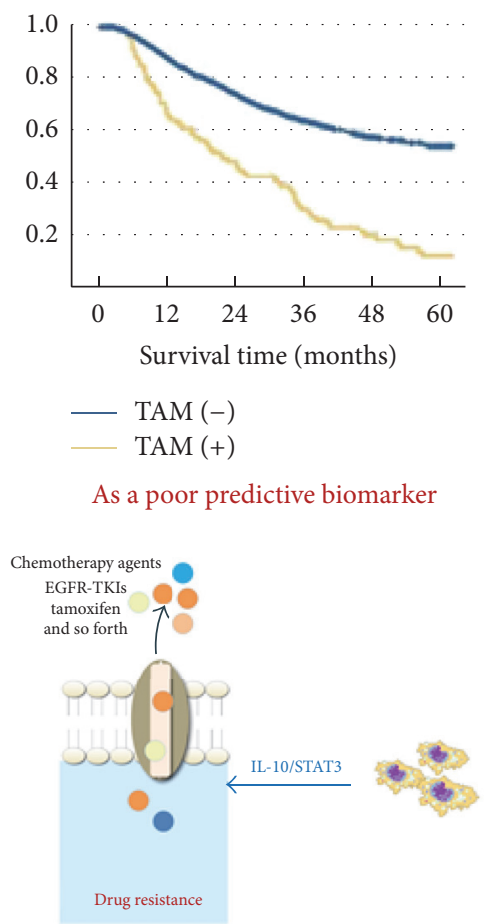

Reducing the curative effect of chemotherapy

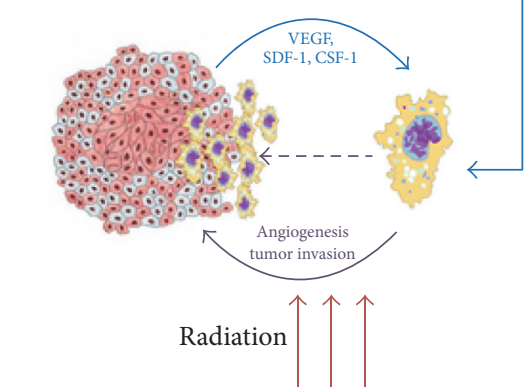

Causing an unfavorable outcome of radiotherapy

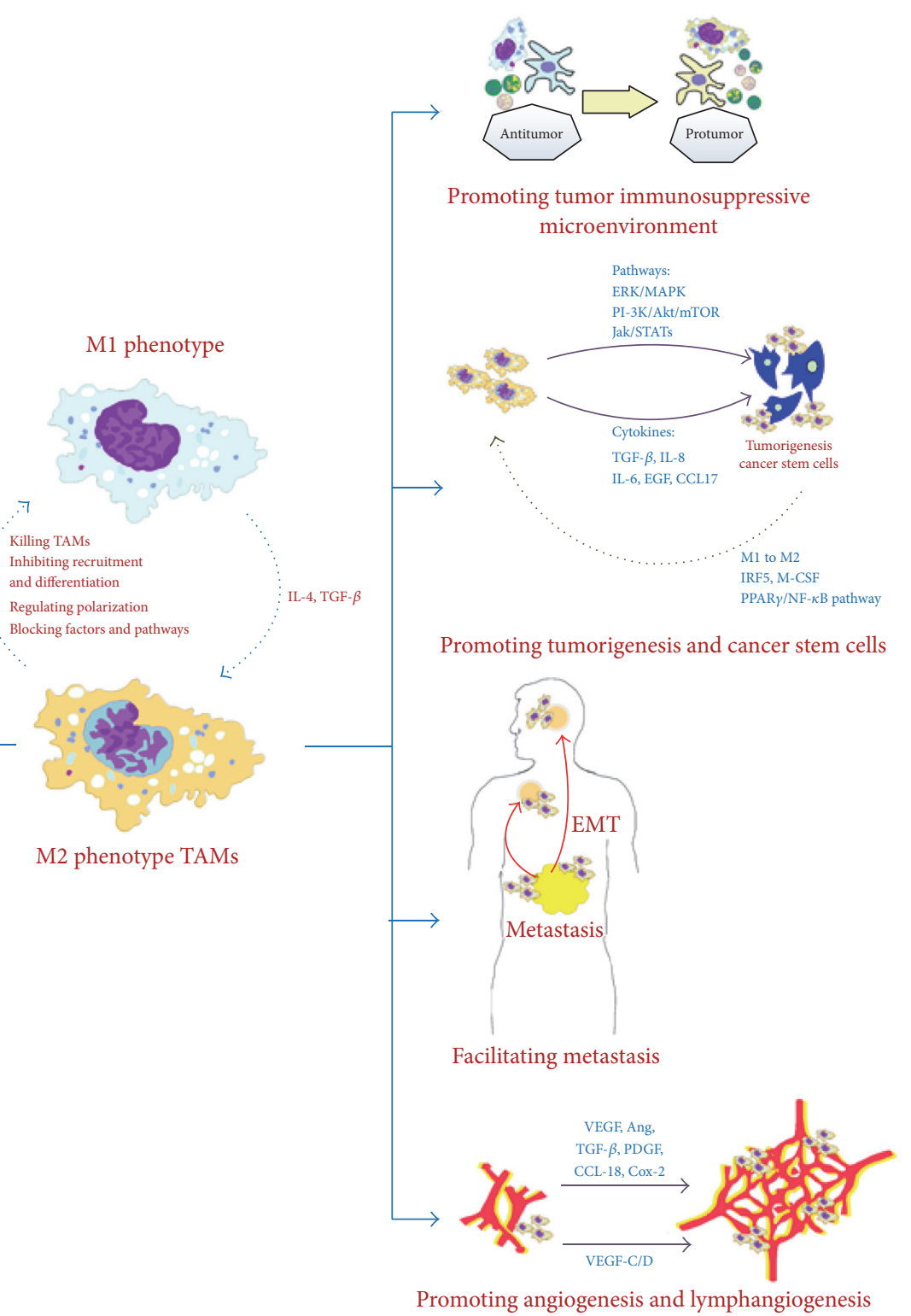

FIGURE 1: TAMs promote tumor progression.

the tumor microenvironment $[1,75]$. Unlike Th1 and Th2 cells, M1 and M2 macrophages are not stably differentiated subsets and can switch the phenotype [131]. In this case, TAMs represent an ideal therapeutic target for blocking tumor progression after being reprogrammed and repolarized to express a proimmunity (M1-like) phenotype [132]. Dimethyl sulfoxide can suppress mouse $4 \mathrm{~T} 1$ breast cancer growth by modulating TAM differentiation [133]. It was found that PA-MSHA can reeducate $\mathrm{CD}_{163}{ }^{+}$TAMs into M1 macrophages through the TLR4-mediated pathway in MPE [27]. M-CSFR signaling was found to govern the phenotype of M2-like MHC-II ${ }^{\text {lo }}$ TAMs, and its blockade results in preferential differentiation of monocytes into M1-like MHCII $^{\text {hi }}$ TAMs [134]. Interferon $\gamma$ and celecoxib can inhibit lung tumor growth by modulating the M2/M1 macrophage ratio in the tumor microenvironment [135].
4.3. Blocking of Factors and Pathways Associated with the Protumor Function of TAMs. The ability of TAMs to accelerate vessel growth is mediated by increased secretion of several proangiogenic factors. Therapeutic success in blocking these protumor activities in preclinical models and early clinical trials highlighted macrophages as effective targets of combination cancer therapy [136]. It is reported that somatostatin derivate (smsDX) can attenuate the TAM-stimulated proliferation, migration, and invasiveness of prostate cancer via NF$\kappa \mathrm{B}$ regulation [137]. Luteolin can also inhibit recruitment of monocytes and migration of Lewis lung carcinoma cells by suppressing chemokine (C-C motif) ligand 2 expression in TAMs [138]. Besides, it has been shown that a cannabinoid receptor 2 agonist inhibits macrophage-induced EMT in non-small cell lung cancer via downregulation of the EGFR pathway [69]. 


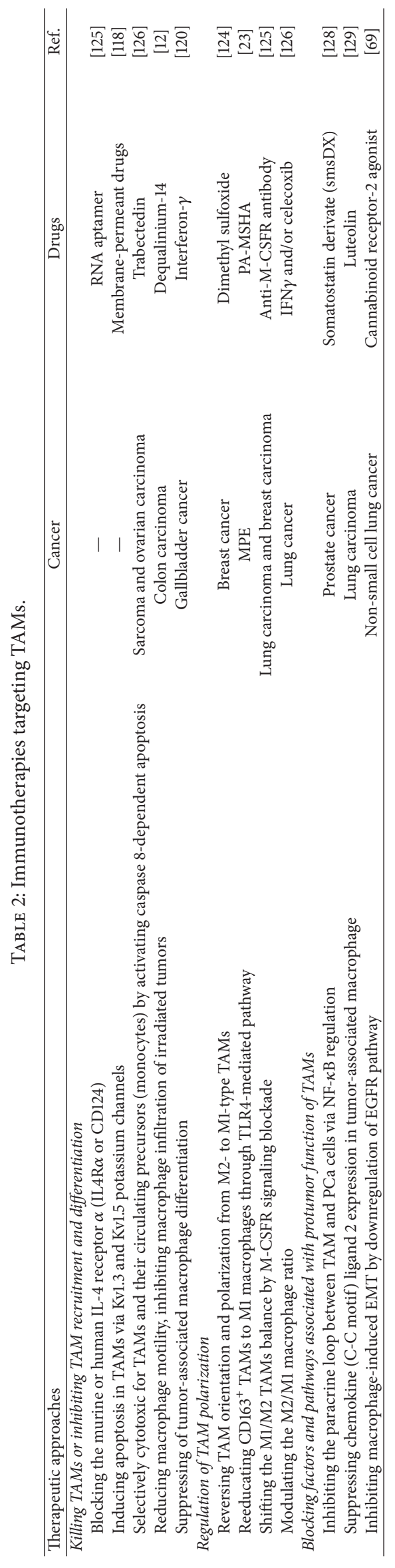




\section{Conclusions}

TAMs comprise an important part of the tumor microenvironment, and their mobilization into the tumor microenvironment plays a key role in malignant progression. Studies have shown that TAMs lead to a poor clinical prognosis and promote progression of various tumors; these cells also correlate with an unfavorable outcome following therapy. After accumulation in tumor tissues, TAMs can remodel the tumor microenvironment to promote matrix remodeling and promote tumor growth and increase angiogenesis CSC-associated tumor progression. With rapid progress in the understanding of TAM functions, new therapeutic approaches against tumors have been developed, such as inhibition of TAM recruitment or suppression of TAM survival, regulation of TAM polarization, reprogramming of TAMs into the antitumor M1 phenotype, and blocking of factors and pathways associated with the protumor function of TAMs. Thus, the increasing knowledge about the biological effects of TAMs and the tumor microenvironment may lead to novel cancer therapies.

\section{Disclosure}

The funders had no role in the study design, data collection and analysis, decision to publish, or preparation of the manuscript.

\section{Competing Interests}

The authors declare that they have no competing interests.

\section{Authors' Contributions}

Qiujun Guo, Zhichao Jin, and Yuan Yuan contributed equally to this work.

\section{Acknowledgments}

This work was supported by the National Twelfth FiveYear Plan for Science and Technology Support Program of China (no. 2014BAI10B01) and the National Natural Science Foundation of China (nos. 81202656, 81273718, 81403346, and 81603610).

\section{References}

[1] V. Gocheva, H.-W. Wang, B. B. Gadea et al., "IL-4 induces cathepsin protease activity in tumor-associated macrophages to promote cancer growth and invasion," Genes and Development, vol. 24, no. 3, pp. 241-255, 2010.

[2] A. Sica, T. Schioppa, A. Mantovani, and P. Allavena, “Tumourassociated macrophages are a distinct M2 polarised population promoting tumour progression: potential targets of anti-cancer therapy," European Journal of Cancer, vol. 42, no. 6, pp. 717-727, 2006.

[3] R. Wang, J. Zhang, S. Chen et al., "Tumor-associated macrophages provide a suitable microenvironment for non-small lung cancer invasion and progression," Lung Cancer, vol. 74, no. 2, pp. 188-196, 2011.
[4] S. Edin, M. L. Wikberg, P.-A. Oldenborg, and R. Palmqvist, "Macrophages: good guys in colorectal cancer," OncoImmunology, vol. 2, no. 2, article e23038, 2013.

[5] T. K. Owonikoko, S. E. Dahlberg, S. A. Khan et al., "A phase 1 safety study of veliparib combined with cisplatin and etoposide in extensive stage small cell lung cancer: a trial of the ECOGACRIN Cancer Research Group (E2511)," Lung Cancer, vol. 89, no. 1, pp. 66-70, 2015.

[6] W. H. Wilson, "Treatment strategies for aggressive lymphomas: what works?” Hematology, vol. 2013, no. 1, pp. 584-590, 2013.

[7] D. Li and E. M. O'Reilly, "Adjuvant and neoadjuvant systemic therapy for pancreas adenocarcinoma," Seminars in Oncology, vol. 42, no. 1, pp. 134-143, 2015.

[8] K. Sugimura, H. Miyata, K. Tanaka et al., "High infiltration of tumor-associated macrophages is associated with a poor response to chemotherapy and poor prognosis of patients undergoing neoadjuvant chemotherapy for esophageal cancer," Journal of Surgical Oncology, vol. 111, no. 6, pp. 752-759, 2015.

[9] B. X. Pei, B. S. Sun, Z. F. Zhang, A. L. Wang, and P. Ren, "Interstitial tumor-associated macrophages combined with tumorderived colony-stimulating factor-1 and interleukin-6, a novel prognostic biomarker in non-small cell lung cancer," Journal of Thoracic \& Cardiovascular Surgery, vol. 148, no. 4, pp. 1208-1216, 2014.

[10] M. Amit and Z. Gil, "Macrophages increase the resistance of pancreatic adenocarcinoma cells to gemcitabine by upregulating cytidine deaminase," OncoImmunology, vol. 2, no. 12, Article ID e27231, 2013.

[11] C. Yang, L. He, P. He et al., "Increased drug resistance in breast cancer by tumor-associated macrophages through IL-10/ STAT3/bcl-2 signaling pathway," Medical Oncology, vol. 32, no. 2, pp. 1-8, 2015.

[12] Q.-J. Xuan, J.-X. Wang, A. Nanding et al., "Tumor-associated macrophages are correlated with tamoxifen resistance in the postmenopausal breast cancer patients," Pathology and Oncology Research, vol. 20, no. 3, pp. 619-624, 2014.

[13] F.-T. Chung, K.-Y. Lee, C.-W. Wang et al., “Tumor-associated macrophages correlate with response to epidermal growth factor receptor-tyrosine kinase inhibitors in advanced nonsmall cell lung cancer," International Journal of Cancer, vol. 131, no. 3, pp. E227-E235, 2012.

[14] J. S. Russell and J. M. Brown, "The irradiated tumor microenvironment: role of tumor-associated macrophages in vascular recovery," Frontiers in Physiology, vol. 4, article 157, 2013.

[15] M. Timaner, R. Bril, O. Kaidar-Person et al., "Dequalinium blocks macrophage-induced metastasis following local radiation," Oncotarget, vol. 6, no. 29, pp. 27537-27554, 2015.

[16] T. A. Wynn, A. Chawla, and J. W. Pollard, "Macrophage biology in development, homeostasis and disease," Nature, vol. 496, no. 7446, pp. 445-455, 2013.

[17] L. Milas, J. Wike, N. Hunter, J. Volpe, and I. Basic, "Macrophage content of murine sarcomas and carcinomas: associations with tumor growth parameters and tumor radiocurability," Cancer Research, vol. 47, no. 4, pp. 1069-1075, 1987.

[18] S. L. Shiao, B. Ruffell, D. G. DeNardo, B. A. Faddegon, C. C. Park, and L. M. Coussens, " $\mathrm{TH}_{2}$-polarized $\mathrm{CD}_{4}^{+} \mathrm{T}$ cells and macrophages limit efficacy of radiotherapy," Cancer Immunology Research, vol. 3, no. 5, pp. 518-525, 2015.

[19] C. Guo, A. Buranych, D. Sarkar, P. B. Fisher, and X. Y. Wang, "The role of tumor-associated macrophages in tumor vascularization," Vasc Cell, vol. 15, no. 8, pp. 917-927, 2013. 
[20] A. Teresa Pinto, M. Laranjeiro Pinto, A. Patrícia Cardoso et al., "Ionizing radiation modulates human macrophages towards a pro-inflammatory phenotype preserving their pro-invasive and pro-angiogenic capacities," Scientific Reports, vol. 6, Article ID 18765, 2016.

[21] C. Dong, M. He, W. Tu et al., "The differential role of human macrophage in triggering secondary bystander effects after either gamma-ray or carbon beam irradiation," Cancer Letters, vol. 363, no. 1, pp. 92-100, 2015.

[22] C.-S. Chiang, S. Y. Fu, S.-C. Wang et al., "Irradiation promotes an M2 macrophage phenotype in tumor hypoxia," Frontiers in Oncology, vol. 2, article 89, 2012.

[23] J. Xu, J. Escamilla, S. Mok et al., "CSF1R signaling blockade stanches tumor-infiltrating myeloid cells and improves the efficacy of radiotherapy in prostate cancer," Cancer Research, vol. 73, no. 9, pp. 2782-2794, 2013.

[24] E. I. Ager, S. V. Kozin, N. D. Kirkpatrick et al., "Blockade of MMP14 activity in murine breast carcinomas: implications for macrophages, vessels, and radiotherapy," Journal of the National Cancer Institute, vol. 107, no. 4, 2015.

[25] J. M. Gwak, M. H. Jang, D. I. Kim, A. N. Seo, and S. Y. Park, "Prognostic value of tumor-associated macrophages according to histologic locations and hormone receptor status in breast cancer," PLoS ONE, vol. 10, no. 4, Article ID e0125728, 2015.

[26] Z.-Y. Yuan, R.-Z. Luo, R.-J. Peng, S.-S. Wang, and C. Xue, "High infiltration of tumor-associated macrophages in triplenegative breast cancer is associated with a higher risk of distant metastasis," OncoTargets and Therapy, vol. 7, pp. 1475-1480, 2014.

[27] C.-N. Lin, C.-J. Wang, Y.-J. Chao, M.-D. Lai, and Y.-S. Shan, "The significance of the co-existence of osteopontin and tumorassociated macrophages in gastric cancer progression," $B M C$ Cancer, vol. 15, no. 1, article 128, 2015.

[28] L. Yang, F. Wang, L. Wang et al., "CD163+ tumor-associated macrophage is a prognostic biomarker and is associated with therapeutic effect on malignant pleural effusion of lung cancer patients," Oncotarget, vol. 6, no. 12, pp. 10592-10603, 2015.

[29] Y. Li, B.-S. Sun, B. Pei et al., "Osteopontin-expressing macrophages in non-small cell lung cancer predict survival," Annals of Thoracic Surgery, vol. 99, no. 4, pp. 1140-1148, 2015.

[30] Y.-H. Ni, L. Ding, X.-F. Huang, Y.-C. Dong, Q.-G. Hu, and Y.-Y. Hou, "Microlocalization of $\mathrm{CD}^{+} 8^{+}$tumor-associated macrophages in tumor stroma correlated with poor clinical outcomes in oral squamous cell carcinoma patients," Tumor Biology, vol. 36, no. 7, pp. 5291-5298, 2015.

[31] K.-F. He, L. Zhang, C.-F. Huang et al., "CD163 ${ }^{+}$tumor-associated macrophages correlated with poor prognosis and cancer stem cells in oral squamous cell carcinoma," BioMed Research International, vol. 2014, Article ID 838632, 9 pages, 2014.

[32] S. Wang, M. Sun, C. Gu et al., "Expression of CD163, interleukin-10, and interferon-gamma in oral squamous cell carcinoma: mutual relationships and prognostic implications," European Journal of Oral Sciences, vol. 122, no. 3, pp. 202-209, 2014.

[33] I. H. Wei, C. M. Harmon, M. Arcerito, D. F. Cheng, R. M. Minter, and D. M. Simeone, "Tumor-associated macrophages are a useful biomarker to predict recurrence after surgical resection of nonfunctional pancreatic neuroendocrine tumors," Annals of Surgery, vol. 260, no. 6, pp. 1088-1094, 2014.

[34] P. Ding, W. Wang, J. Wang, Z. Yang, and L. Xue, "Expression of tumor-associated macrophage in progression of human glioma," Cell Biochemistry and Biophysics, vol. 70, no. 3, pp. 1625-1631, 2014.
[35] M. Illemann, O. D. Laerum, J. P. Hasselby et al., "Urokinase-type plasminogen activator receptor (UPAR) on tumor-associated macrophages is a marker of poor prognosis in colorectal cancer," Cancer Medicine, vol. 3, no. 4, pp. 855-864, 2014.

[36] M. Kinouchi, K. Miura, T. Mizoi et al., "Infiltration of CD40-positive tumor-associated macrophages indicates a favorable prognosis in colorectal cancer patients," HepatoGastroenterology, vol. 60, no. 121, pp. 83-88, 2013.

[37] Y.-C. Hou, Y.-J. Chao, H.-L. Tung, H.-C. Wang, and Y.-S. Shan, "Coexpression of CD44-positive/CD133-positive cancer stem cells and CD204-positive tumor-associated macrophages is a predictor of survival in pancreatic ductal adenocarcinoma," Cancer, vol. 120, no. 17, pp. 2766-2777, 2014.

[38] C. Lan, X. Huang, S. Lin et al., "Expression of M2-polarized macrophages is associated with poor prognosis for advanced epithelial ovarian cancer," Technology in Cancer Research \& Treatment, vol. 12, no. 3, pp. 259-267, 2013.

[39] H. Takayama, K. Nishimura, A. Tsujimura et al., "Increased infiltration of tumor associated macrophages is associated with poor prognosis of bladder carcinoma in situ after intravesical bacillus calmette-guerin instillation," Journal of Urology, vol. 181, no. 4, pp. 1894-1900, 2009.

[40] X.-F. Jiang, Q.-L. Tang, X.-M. Shen et al., "Tumor-associated macrophages, epidermal growth factor receptor correlated with the triple negative phenotype in endometrial endometrioid adenocarcinoma," Pathology Research and Practice, vol. 208, no. 12, pp. 730-735, 2012.

[41] K. Gollapudi, C. Galet, T. Grogan et al., "Association between tumor-associated macrophage infiltration, high grade prostate cancer, and biochemical recurrence after radical prostatectomy," American Journal of Cancer Research, vol. 3, no. 5, pp. 523-529, 2013.

[42] W. Hu, Y. Qian, F. Yu et al., "Alternatively activated macrophages are associated with metastasis and poor prognosis in prostate adenocarcinoma," Oncology Letters, vol. 10, no. 3, pp. 1390-1396, 2015.

[43] D. W. Scott and C. Steidl, "The classical Hodgkin lymphoma tumor microenvironment: macrophages and gene expressionbased modeling," Hematology, vol. 2014, no. 1, pp. 144-150, 2014.

[44] T. Sasayama, K. Tanaka, T. Mizowaki et al., "Tumor-associated macrophages associate with cerebrospinal fluid interleukin-10 and survival in Primary Central Nervous System Lymphoma (PCNSL)," Brain Pathology, vol. 26, no. 4, pp. 479-487, 2016.

[45] W. Zhang, L. Wang, D. Zhou, Q. Cui, D. Zhao, and Y. Wu, "Expression of tumor-associated macrophages and vascular endothelial growth factor correlates with poor prognosis of peripheral T-cell lymphoma, not otherwise specified," Leukemia and Lymphoma, vol. 52, no. 1, pp. 46-52, 2011.

[46] Q.-C. Cai, H. Liao, S.-X. Lin et al., "High expression of tumorinfiltrating macrophages correlates with poor prognosis in patients with diffuse large B-cell lymphoma," Medical Oncology, vol. 29, no. 4, pp. 2317-2322, 2012.

[47] Q. Guo, J. Li, and H. Lin, "Effect and molecular mechanisms of traditional Chinese medicine on regulating tumor immunosuppressive microenvironment," BioMed Research International, vol. 2015, Article ID 261620, 12 pages, 2015.

[48] J. Kim and J.-S. Bae, "Tumor-associated macrophages and neutrophils in tumor microenvironment," Mediators of Inflammation, vol. 2016, Article ID 6058147, 11 pages, 2016.

[49] N. L. Costa, M. C. Valadares, P. P. C. Souza et al., "Tumorassociated macrophages and the profile of inflammatory 
cytokines in oral squamous cell carcinoma," Oral Oncology, vol. 49, no. 3, pp. 216-223, 2013.

[50] Q. Han, H. Shi, and F. Liu, “CD163+ M2-type tumor-associated macrophage support the suppression of tumor-infiltrating $\mathrm{T}$ cells in osteosarcoma," International Immunopharmacology, vol. 34, pp. 101-106, 2016.

[51] T. Krneta, A. Gillgrass, and A. A. Ashkar, "The influence of macrophages and the tumor microenvironment on natural killer cells," Current Molecular Medicine, vol. 13, no. 1, pp. 6879, 2013.

[52] Q.-J. Guo and J. Li, “The role of tumor-associated macrophages in remodeling tumor immune microenvironment," Tumor, vol. 33, no. 10, pp. 922-927, 2013.

[53] M. C. Schmid and J. A. Varner, "Myeloid cells in tumor inflammation," Vascular Cell, vol. 4, article no. 14, 2012.

[54] M. Lohela, A.-J. Casbon, A. Olow et al., "Intravital imaging reveals distinct responses of depleting dynamic tumorassociated macrophage and dendritic cell subpopulations," Proceedings of the National Academy of Sciences of the United States of America, vol. 111, no. 47, pp. E5086-E5095, 2014.

[55] P. C. Rodriguez, D. G. Quiceno, J. Zabaleta et al., "Arginase I production in the tumor microenvironment by mature myeloid cells inhibits T-cell receptor expression and antigen-specific Tcell responses," Cancer Research, vol. 64, no. 16, pp. 5839-5849, 2004.

[56] T. Lu, R. Ramakrishnan, S. Altiok et al., "Tumor-infiltrating myeloid cells induce tumor cell resistance to cytotoxic T cells in mice," Journal of Clinical Investigation, vol. 121, no. 10, pp. 40154029, 2011.

[57] A. Kondo, T. Yamashita, H. Tamura et al., "Interferon- $\gamma$ and tumor necrosis factor- $\alpha$ induce an immunoinhibitory molecule, $\mathrm{B} 7-\mathrm{H} 1$, via nuclear factor- $\kappa \mathrm{B}$ activation in blasts in myelodysplastic syndromes," Blood, vol. 116, no. 7, pp. 1124-1131, 2010.

[58] S. I. Grivennikov and M. Karin, "Inflammatory cytokines in cancer: tumour necrosis factor and interleukin 6 take the stage," Annals of the Rheumatic Diseases, vol. 70, no. 1, pp. i104-i108, 2011.

[59] D. M. Mosser and J. P. Edwards, "Exploring the full spectrum of macrophage activation," Nature Reviews Immunology, vol. 8, no. 12, pp. 958-969, 2008.

[60] F. Balkwill, K. A. Charles, and A. Mantovani, "Smoldering and polarized inflammation in the initiation and promotion of malignant disease," Cancer Cell, vol. 7, no. 3, pp. 211-217, 2005.

[61] F. R. Balkwill and A. Mantovani, "Cancer-related inflammation: common themes and therapeutic opportunities," Seminars in Cancer Biology, vol. 22, no. 1, pp. 33-40, 2012.

[62] L. M. Coussens and Z. Werb, "Inflammation and cancer," Nature, vol. 420, no. 6917, pp. 860-867, 2002.

[63] S. I. Grivennikov, F. R. Greten, and M. Karin, "Immunity, inflammation, and cancer," Cell, vol. 140, no. 6, pp. 883-899, 2010.

[64] H. A. Smith and Y. Kang, "The metastasis-promoting roles of tumor-associated immune cells," Journal of Molecular Medicine, vol. 91, no. 4, pp. 411-429, 2013.

[65] J. Condeelis and J. W. Pollard, "Macrophages: obligate partners for tumor cell migration, invasion, and metastasis," Cell, vol. 124, no. 2, pp. 263-266, 2006.

[66] H. Wang, X. Wang, X. Li et al., "CD68 ${ }^{+} \mathrm{HLA}^{-D R^{+}}$M1-like macrophages promote motility of HCC cells via NF- $\kappa \mathrm{B} / \mathrm{FAK}$ pathway," Cancer Letters, vol. 345, no. 1, pp. 91-99, 2014.
[67] T. H. Xu, L. Wei, Y. Zhang et al., “Tumor-associated macrophage-derived IL- 6 and IL- 8 enhance invasive activity of LoVo cells induced by PRL-3 in a KCNN4 channel-dependent manner," Bmc Cancer, vol. 14, no. 5, pp. 697-710, 2014.

[68] B.-Z. Qian, J. Li, H. Zhang et al., "CCL2 recruits inflammatory monocytes to facilitate breast-tumour metastasis," Nature, vol. 475, no. 7355, pp. 222-225, 2011.

[69] T. Kitamura, B.-Z. Qian, D. Soong et al., "CCL2-induced chemokine cascade promotes breast cancer metastasis by enhancing retention of metastasis-associated macrophages," Journal of Experimental Medicine, vol. 212, no. 7, pp. 1043-1059, 2015.

[70] Q. Chen, X. H.-F. Zhang, and J. Massagué, "Macrophage binding to receptor VCAM-1 transmits survival signals in breast cancer cells that invade the lungs," Cancer Cell, vol. 20, no. 4, pp. 538549, 2011.

[71] B. D. Robinson, G. L. Sica, Y.-F. Liu et al., "Tumor microenvironment of metastasis in human breast carcinoma: a potential prognostic marker linked to hematogenous dissemination," Clinical Cancer Research, vol. 15, no. 7, pp. 2433-2441, 2009.

[72] J. P. Thiery, H. Acloque, R. Y. J. Huang, and M. A. Nieto, "Epithelial-mesenchymal transitions in development and disease," Cell, vol. 139, no. 5, pp. 871-890, 2009.

[73] D. K. Gupta, N. Singh, and D. K. Sahu, “TGF- $\beta$ mediated crosstalk between malignant hepatocyte and tumor microenvironment in hepatocellular carcinoma," Cancer Growth o Metastasis, vol. 7, no. 7, pp. 1-8, 2014.

[74] M. De Palma and C. E. Lewis, "Macrophage regulation of tumor responses to anticancer therapies," Cancer Cell, vol. 23, no. 3, pp. 277-286, 2013.

[75] C.-Y. Liu, J.-Y. Xu, X.-Y. Shi et al., "M2-polarized tumorassociated macrophages promoted epithelial-mesenchymal transition in pancreatic cancer cells, partially through TLR4/IL10 signaling pathway," Laboratory Investigation, vol. 93, no. 7, pp. 844-854, 2013.

[76] J. Ravi, M. Elbaz, N. A. Wani, M. W. Nasser, and R. K. Ganju, "Cannabinoid receptor-2 agonist inhibits macrophage induced EMT in non-small cell lung cancer by downregulation of EGFR pathway," Molecular Carcinogenesis, 2016.

[77] L. Lin, Y.-S. Chen, Y.-D. Yao et al., "CCL18 from tumorassociated macrophages promotes angio-genesis in breast cancer," Oncotarget, vol. 6, no. 33, pp. 34758-34773, 2015.

[78] B. R. Zetter, "Angiogenesis and tumor metastasis," Annual Review of Medicine, vol. 49, no. 7170, pp. 407-424, 1998.

[79] X. Xia, R. Du, L. Zhao, W. Sun, and X. Wang, "Expression of AEG-1 and microvessel density correlates with metastasis and prognosis of oral squamous cell carcinoma," Human Pathology, vol. 45, no. 4, pp. 858-865, 2014.

[80] S. Karaman and M. Detmar, "Mechanisms of lymphatic metastasis," The Journal of Clinical Investigation, vol. 124, no. 3, pp. 922-928, 2014.

[81] S. A. Stacker, S. P. Williams, T. Karnezis, R. Shayan, S. B. Fox, and M. G. Achen, "Lymphangiogenesis and lymphatic vessel remodelling in cancer," Nature Reviews Cancer, vol. 14, no. 3, pp. 159-172, 2014.

[82] Y. Yang, M. Sun, L. Wang, and B. Jiao, "HIFs, angiogenesis, and cancer," Journal of Cellular Biochemistry, vol. 114, no. 5, pp. 967974, 2013.

[83] J. M. Breuss and P. Uhrin, "VEGF-initiated angiogenesis and the uPA/uPAR system," Cell Adhesion and Migration, vol. 6, no. 6, pp. 535-540, 2012. 
[84] D. P. Toomey, J. F. Murphy, and K. C. Conlon, "COX-2, VEGF and tumour angiogenesis," Surgeon Journal of the Royal Colleges of Surgeons of Edinburgh \& Ireland, vol. 7, no. 7, pp. 174-180, 2009.

[85] I. F. Dunn, O. Heese, and P. M. Black, "Growth factors in glioma angiogenesis: FGFs, PDGF, EGF, and TGFs," Journal of NeuroOncology, vol. 50, no. 1-2, pp. 121-137, 2000.

[86] E. Fagiani and G. Christofori, "Angiopoietins in angiogenesis," Cancer Letters, vol. 328, no. 1, pp. 18-26, 2013.

[87] E.-J. Yeo, L. Cassetta, B.-Z. Qian et al., "Myeloid wnt7b mediates the angiogenic switch and metastasis in breast cancer," Cancer Research, vol. 74, no. 11, pp. 2962-2973, 2014.

[88] E. I. Deryugina, E. Zajac, A. Juncker-Jensen, T. A. Kupriyanova, L. Welter, and J. P. Quigley, "Tissue-infiltrating neutrophils constitute the major in vivo source of angiogenesis-inducing MMP-9 in the tumor microenvironment," Neoplasia, vol. 16, no. 10, pp. 771-788, 2014.

[89] G. Bergers and L. E. Benjamin, "Tumorigenesis and the angiogenic switch," Nature Reviews Cancer, vol. 3, no. 6, pp. 401-410, 2003.

[90] E. I. Deryugina and J. P. Quigley, "Pleiotropic roles of matrix metalloproteinases in tumor angiogenesis: contrasting, overlapping and compensatory functions," Biochimica et Biophysica Acta-Molecular Cell Research, vol. 1803, no. 1, pp. 103-120, 2010.

[91] A. Casazza, D. Laoui, M. Wenes et al., "Impeding macrophage entry into hypoxic tumor areas by Sema3A/Nrpl signaling blockade inhibits angiogenesis and restores antitumor immunity," Cancer Cell, vol. 24, no. 6, pp. 695-709, 2013.

[92] L. J. Mu, J. S. Wang, Y. Z. Chen et al., "Hypoxia-inducible factor$1 \alpha$ and semaphorin $4 d$ genes involved with tumor-associated macrophage-induced metastatic behavior and clinical significance in colon cancer," Chinese Medical Journal, vol. 127, no. 20, pp. 3568-3575, 2014.

[93] D. Laoui, E. Van Overmeire, G. Di Conza et al., "Tumor hypoxia does not drive differentiation of tumor-associated macrophages but rather fine-tunes the M2-like macrophage population," Cancer Research, vol. 74, no. 1, pp. 24-30, 2014.

[94] L. D. E. Jensen, R. Cao, E.-M. Hedlund et al., "Nitric oxide permits hypoxia-induced lymphatic perfusion by controlling arterial-lymphatic conduits in zebrafish and glass catfish," Proceedings of the National Academy of Sciences of the United States of America, vol. 106, no. 43, pp. 18408-18413, 2009.

[95] S. Liao and T. P. Padera, "Lymphatic function and immune regulation in health and disease," Lymphatic Research and Biology, vol. 11, no. 3, pp. 136-143, 2013.

[96] S. G. Rockson, "Lymphatics: where the circulation meets the immune system," Lymphatic Research \& Biology, vol. 11, no. 3, p. 115, 2013.

[97] J. Wang, Y. Guo, B. Wang et al., "Lymphatic microvessel density and vascular endothelial growth factor-C and -D as prognostic factors in breast cancer: a systematic review and meta-analysis of the literature," Molecular Biology Reports, vol. 39, no. 12, pp. 11153-11165, 2012.

[98] Y. Chen, J. Yan, Z. Wang et al., "A meta-analysis of the relationship between lymphatic microvessel density and the survival of patient with colorectal cancer," Lymphology, vol. 46, no. 1, pp. 42-51, 2013.

[99] I. Pastushenko, P. B. Vermeulen, F. J. Carapeto et al., "Blood microvessel density, lymphatic microvessel density and lymphatic invasion in predicting melanoma metastases: systematic review and meta-analysis," British Journal of Dermatology, vol. 170, no. 1, pp. 66-77, 2014.
[100] J. Wang, K. Li, B. Wang, and J. Bi, "Lymphatic microvessel density as a prognostic factor in non-small cell lung carcinoma: a meta-analysis of the literature," Molecular Biology Reports, vol. 39, no. 5, pp. 5331-5338, 2012.

[101] S. F. Schoppmann, A. Fenzl, K. Nagy et al., "VEGF-C expressing tumor-associated macrophages in lymph node positive breast cancer: impact on lymphangiogenesis and survival," Surgery, vol. 139, no. 6, pp. 839-846, 2006.

[102] B. Zhang, G. Yao, Y. Zhang et al., "M2-Polarized tumor-associated macrophages are associated with poor prognoses resulting from accelerated lymphangiogenesis in lung adenocarcinoma," Clinics, vol. 66, no. 11, pp. 1879-1886, 2011.

[103] S. F. Schoppmann, P. Birner, J. Stöckl et al., “Tumor-associated macrophages express lymphatic endothelial growth factors and are related to peritumoral lymphangiogenesis," The American Journal of Pathology, vol. 161, no. 3, pp. 947-956, 2002.

[104] Z. Špirić, Ž. Eri, and M. Erić, "Significance of vascular endothelial growth factor (VEGF)-C and VEGF-D in the progression of cutaneous melanoma," International Journal of Surgical Pathology, vol. 23, no. 8, pp. 629-637, 2015.

[105] H. Kurahara, S. Takao, K. Maemura et al., "M2-Polarized tumorassociated macrophage infiltration of regional lymph nodes is associated with nodal lymphangiogenesis and occult nodal involvement in pn0 pancreatic cancer," Pancreas, vol. 42, no. 1 , pp. 155-159, 2013.

[106] Y.-F. Sun, Y. Xu, X.-R. Yang et al., "Circulating stem cell-like epithelial cell adhesion molecule-positive tumor cells indicate poor prognosis of hepatocellular carcinoma after curative resection," Hepatology, vol. 57, no. 4, pp. 1458-1468, 2013.

[107] Z. Zhen, J. Ren, and M. O’Neil, “Impact of stem cell marker expression on recurrence of TACE-treated hepatocellular carcinoma post liver transplantation," Bmc Cancer, vol. 12, no. 1, pp. 2604-2615, 2012.

[108] M. Jinushi, S. Chiba, H. Yoshiyama et al., “Tumor-associated macrophages regulate tumorigenicity and anticancer drug responses of cancer stem/initiating cells," Proceedings of the National Academy of Sciences of the United States of America, vol. 108, no. 30, pp. 12425-12430, 2011.

[109] S. Schwitalla, A. A. Fingerle, P. Cammareri et al., "Intestinal tumorigenesis initiated by dedifferentiation and acquisition of stem-cell-like properties," Cell, vol. 152, no. 1-2, pp. 25-38, 2013.

[110] J. Yang, D. Liao, C. Chen et al., "Tumor-associated macrophages regulate murine breast cancer stem cells through a novel paracrine egfr/stat3/sox-2 signaling pathway," Stem Cells, vol. 31, no. 2, pp. 248-258, 2013.

[111] C. Zhang, X. Hu, X. Y. Liu et al., "Effect of tumor-associated macrophages on gastric cancer stem cell in omental milky spots and lymph node micrometastasis," International Journal of Clinical \& Experimental Pathology, vol. 8, no. 11, 2015.

[112] Q.-M. Fan, Y.-Y. Jing, G.-F. Yu et al., "Tumor-associated macrophages promote cancer stem cell-like properties via transforming growth factor-betal-induced epithelial-mesenchymal transition in hepatocellular carcinoma," Cancer Letters, vol. 352, no. 2, pp. 160-168, 2014.

[113] X.-Z. Ye, S.-L. Xu, Y.-H. Xin et al., "Tumor-associated microgl$\mathrm{ia} /$ macrophages enhance the invasion of glioma stem-like cells via TGF- $\beta 1$ signaling pathway," Journal of Immunology, vol. 189, no. 1, pp. 444-453, 2012.

[114] H. Lu, K. R. Clauser, W. L. Tam et al., "A breast cancer stem cell niche supported by juxtacrine signalling from monocytes and macrophages," Nature Cell Biology, vol. 16, no. 11, pp. 1105-1117, 2014. 
[115] X. Deng, P. Zhang, T. Liang, S. Deng, X. Chen, and L. Zhu, "Ovarian cancer stem cells induce the M2 polarization of macrophages through the PPAR $\gamma$ and NF- $\kappa \mathrm{B}$ pathways," International Journal of Molecular Medicine, vol. 36, no. 2, pp. 449454, 2015.

[116] M. Jinushi, "Role of cancer stem cell-associated inflammation in creating pro-inflammatory tumorigenic microenvironments," OncoImmunology, vol. 3, no. 5, Article ID e28862, 2014.

[117] T. Chen, X. Wang, L. Guo et al., "Embryonic stem cells promoting macrophage survival and function are crucial for teratoma development," Frontiers in Immunology, vol. 5, article 275, 2014.

[118] C. D. Mills, L. L. Lenz, and R. A. Harris, "A breakthrough: macrophage-directed cancer immunotherapy," Cancer Research, vol. 76, no. 3, pp. 513-516, 2016.

[119] C. Zhang, L. Gao, Y. Cai et al., "Inhibition of tumor growth and metastasis by photoimmunotherapy targeting tumor-associated macrophage in a sorafenib-resistant tumor model," Innovation \& Social Process, vol. 84, no. 9, pp. 127-136, 2016.

[120] S. Vinogradov, G. Warren, and X. Wei, "Macrophages associated with tumors as potential targets and therapeutic intermediates," Nanomedicine, vol. 9, no. 5, pp. 695-707, 2014.

[121] D. Laoui, E. Van Overmeire, P. De Baetselier, J. A. Van Ginderachter, and G. Raes, "Functional relationship between tumorassociated macrophages and macrophage colony-stimulating factor as contributors to cancer progression," Frontiers in Immunology, vol. 5, no. 5, article 489, 2014.

[122] V. Chitu and E. R. Stanley, "Colony-stimulating factor-1 in immunity and inflammation," Current Opinion in Immunology, vol. 18, no. 1, pp. 39-48, 2006.

[123] S. M. Pyonteck, L. Akkari, A. J. Schuhmacher et al., "CSF-1R inhibition alters macrophage polarization and blocks glioma progression," Nature Medicine, vol. 19, no. 10, pp. 1264-1272, 2013.

[124] C. H. Ries, M. A. Cannarile, S. Hoves et al., "Targeting tumorassociated macrophages with anti-CSF-1R antibody reveals a strategy for cancer therapy," Cancer Cell, vol. 25, no. 6, pp. 846859, 2014.

[125] F. Roth, A. C. De La Fuente, J. L. Vella, A. Zoso, L. Inverardi, and P. Serafini, "Aptamer-mediated blockade of IL4R $\alpha$ triggers apoptosis of MDSCs and limits tumor progression," Cancer Research, vol. 72, no. 6, pp. 1373-1383, 2012.

[126] P. Allavena, G. Germano, C. Belgiovine, M. D'Incalci, and A. Mantovani, "A drug from the sea that strikes tumor-associated macrophages," OncoImmunology, vol. 2, no. 6, article e24614, 2013.

[127] G. Germano, R. Frapolli, C. Belgiovine et al., "Role of macrophage targeting in the antitumor activity of trabectedin," Cancer Cell, vol. 23, no. 2, pp. 249-262, 2013.

[128] L. Leanza, M. Zoratti, E. Gulbins, and I. Szabò, "Induction of apoptosis in macrophages via Kv1.3 and Kv1.5 potassium channels," Current Medicinal Chemistry, vol. 19, no. 31, pp. 53945404, 2012.

[129] R. A. Franklin and M. O. Li, "Ontogeny of tumor-associated macrophages and its implication in cancer regulation," Trends in Cancer, vol. 2, no. 1, pp. 20-34, 2016.

[130] S. Tao, Y. Ye, L. Xiaoguang et al., "Inhibition of tumor angiogenesis by interferon- $\gamma$ by suppression of tumor-associated macrophage differentiation," Oncology Research Featuring Preclinical \& Clinical Cancer Therapeutics, vol. 21, no. 5, pp. 227235, 2014.
[131] A. Mantovani, S. Sozzani, M. Locati, P. Allavena, and A. Sica, "Macrophage polarization: tumor-associated macrophages as a paradigm for polarized M2 mononuclear phagocytes," Trends in Immunology, vol. 23, no. 11, pp. 549-555, 2002.

[132] T. Chanmee, P. Ontong, K. Konno, and N. Itano, “Tumorassociated macrophages as major players in the tumor microenvironment," Cancers, vol. 6, no. 3, pp. 1670-1690, 2014.

[133] R. Deng, S.-M. Wang, T. Yin et al., "Dimethyl sulfoxide suppresses mouse $4 \mathrm{~T} 1$ breast cancer growth by modulating tumor-associated macrophage differentiation," Journal of Breast Cancer, vol. 17, no. 1, pp. 25-32, 2014.

[134] E. Van Overmeire, B. Stijlemans, F. Heymann et al., "M-CSF and GM-CSF receptor signaling differentially regulate monocyte maturation and macrophage polarization in the tumor microenvironment," Cancer Research, vol. 76, no. 1, pp. 35-42, 2016.

[135] F. Ren, M. Fan, J. Mei et al., "Interferon- $\gamma$ and celecoxib inhibit lung-tumor growth through modulating M2/M1 macrophage ratio in the tumor microenvironment," Drug Design, Development and Therapy, vol. 8, pp. 1527-1538, 2014.

[136] R. Noy and J. Pollard, “Tumor-associated macrophages: from mechanisms to therapy," Immunity, vol. 41, no. 1, pp. 49-61, 2014.

[137] Z. Guo, Z. Xing, X. Cheng et al., "Somatostatin derivate (smsDX) attenuates the TAM-stimulated proliferation, migration and invasion of prostate cancer via NF- $\kappa \mathrm{B}$ regulation," PLoS ONE, vol. 10, no. 5, Article ID e0124292, 2015.

[138] H.-J. Choi, H.-J. Choi, T.-W. Chung, and K.-T. Ha, "Luteolin inhibits recruitment of monocytes and migration of Lewis lung carcinoma cells by suppressing chemokine (C-C motif) ligand 2 expression in tumor-associated macrophage," Biochemical and Biophysical Research Communications, vol. 470, no. 1, pp. 101106, 2016. 


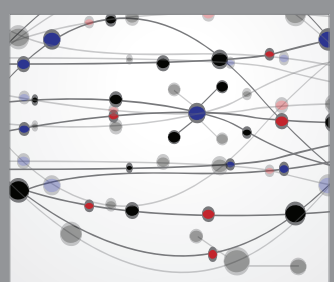

The Scientific World Journal
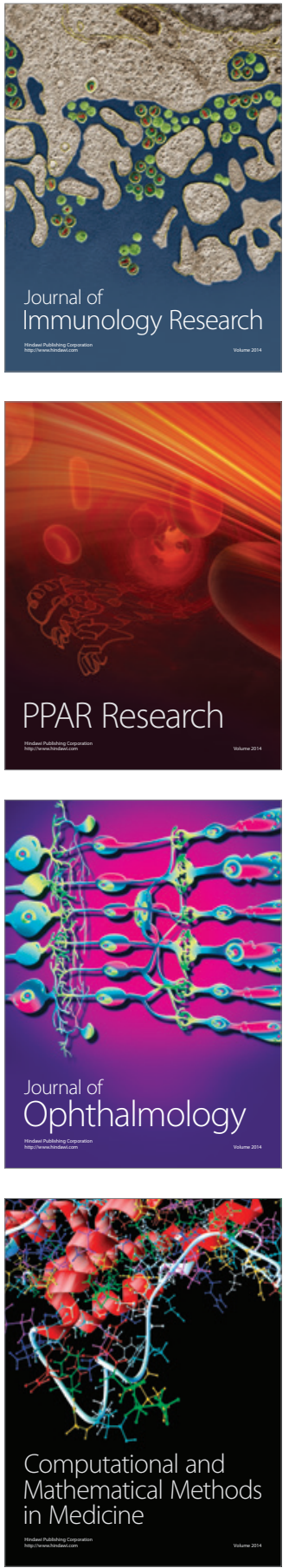

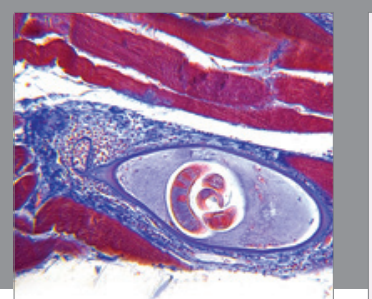

Gastroenterology Research and Practice

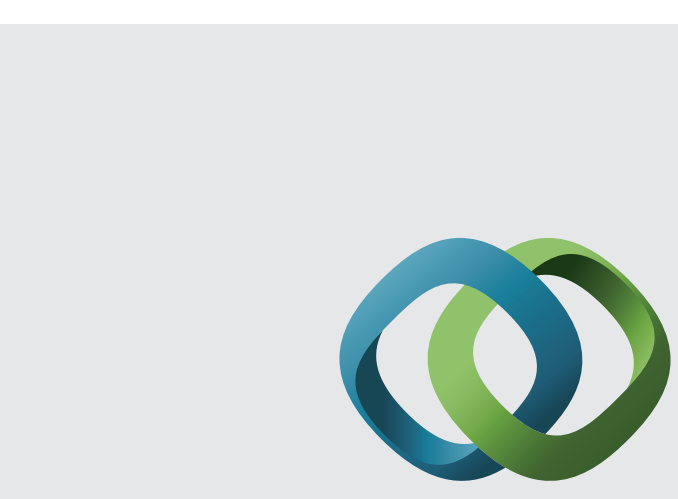

\section{Hindawi}

Submit your manuscripts at

http://www.hindawi.com
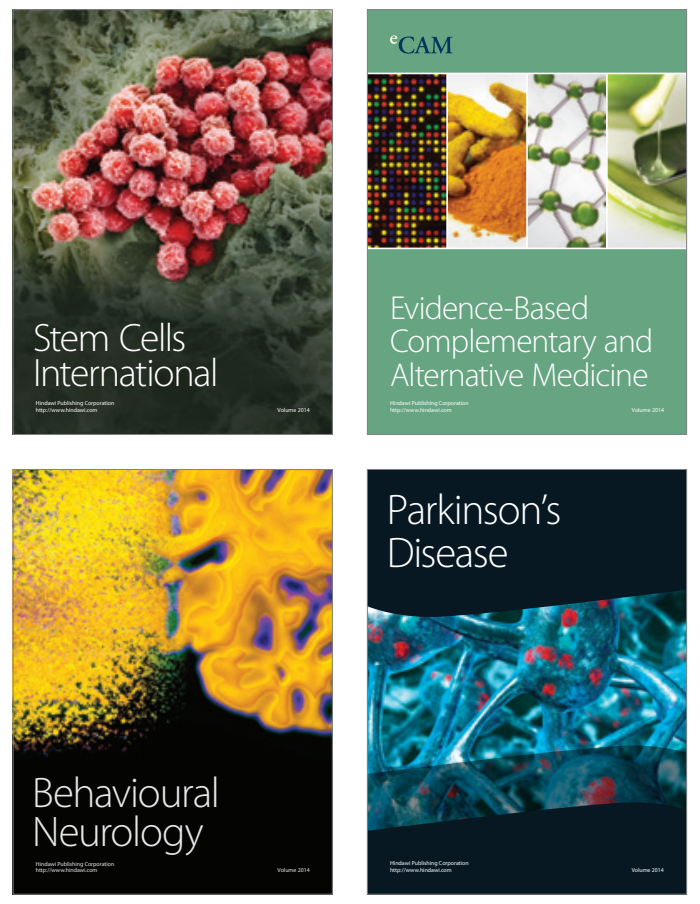
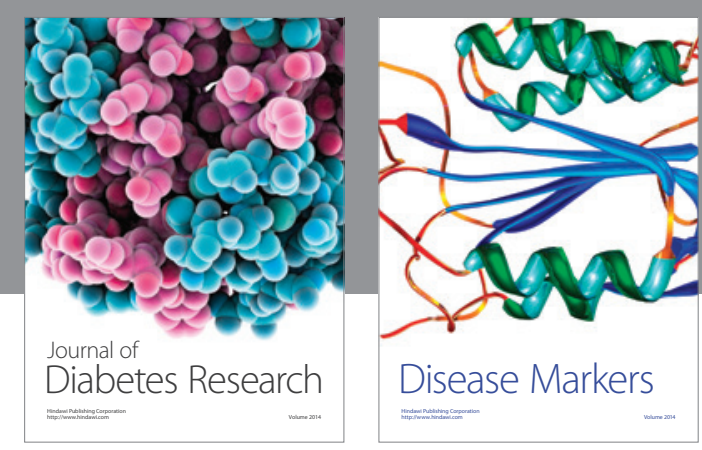

Disease Markers
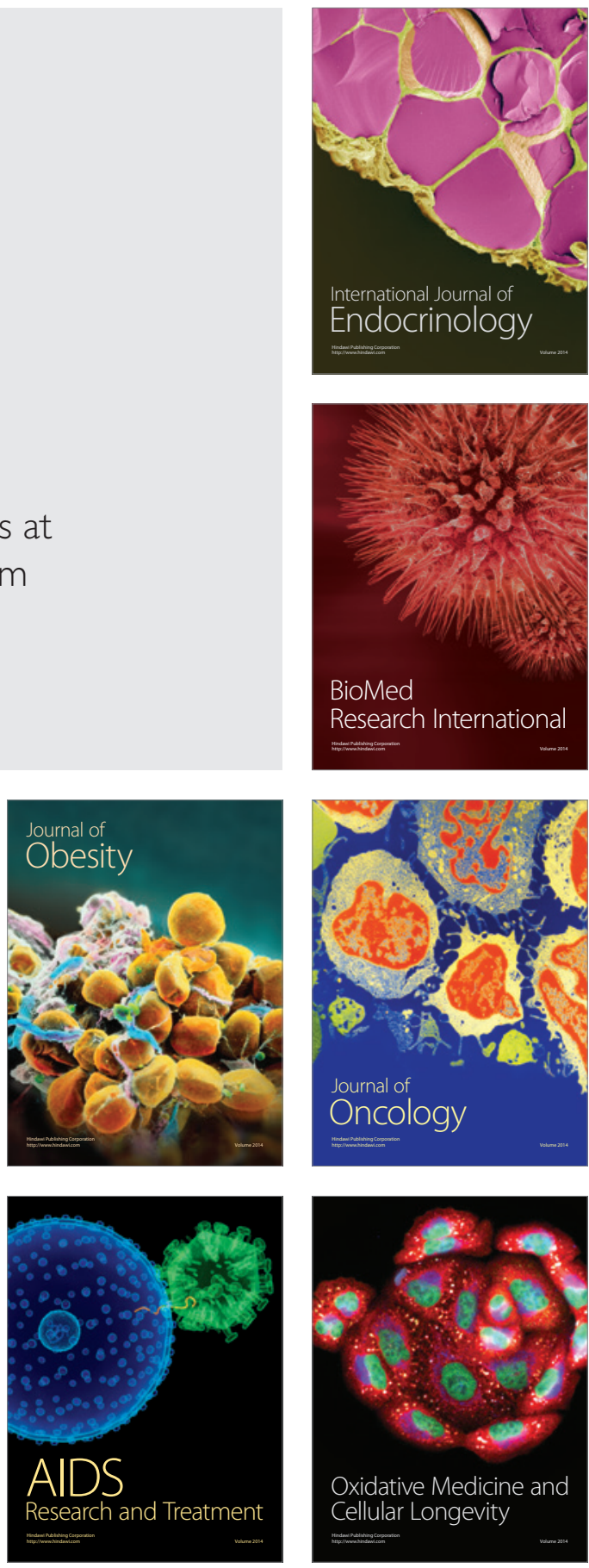